\title{
Neuropsychiatric symptoms in 921 elderly subjects with dementia: a comparison between vascular and neurodegenerative types
}

Caputo M, Monastero R, Mariani E, Santucci A, Mangialasche F, Camarda R, Senin U, Mecocci P. Neuropsychiatric symptoms in 921 elderly subjects with dementia: a comparison between vascular and neurodegenerative types.

Objective: i) to describe the neuropsychiatric profile of elderly subjects with dementia by comparing vascular $(\mathrm{VaD})$ and degenerative dementias, i.e. dementia with Lewy bodies (DLB) and Alzheimer's disease (AD); ii) to assess whether the severity and type of dementia are associated with clinically relevant neuropsychiatric symptoms (CR-NPS).

Method: One hundred and thirty-one out-patients with $\mathrm{VaD}, 100$ with DLB and 690 with AD were studied. NPS were evaluated by the neuropsychiatric inventory (NPI).

Results: Vascular dementia had lower total and domain-specific NPI scores and a lower frequency of CR-NPS than AD and DLB, for which frequency of CR-NPS increased significantly with disease severity, particularly in AD. Logistic regression analysis showed that a higher CDR score and a diagnosis of degenerative dementia were independently associated with CR-NPS.

Conclusion: Vascular dementia is associated less with CR-NPS than AD and DLB. Frequency of CR-NPS increases with disease severity in $\mathrm{AD}$ and, to a lesser extent, in DLB.

\section{Caputo ${ }^{1 *}$, R. Monastero ${ }^{2 *}$, E. Mariani ${ }^{1}$, A. Santucci ${ }^{1}$, F. Mangialasche ${ }^{1}$, R. Camarda ${ }^{2}$, U. Senin ${ }^{1}$, P. Mecocci ${ }^{1}$}

${ }^{1}$ Section of Gerontology and Geriatrics, Department of Clinical and Experimental Medicine, University of Perugia, Perugia, Italy and ${ }^{2}$ Laboratory of Epidemiology and Psychology of Aging and Dementia, Section of Neurology and Psychiatry, Department of Clinical Neuroscience, University of Palermo, Palermo, Italy

Key words: neuropsychiatric symptoms; vascular dementia; dementia with Lewy bodies; Alzheimer's disease; dementia severity

Patrizia Mecocci MD, PhD, Institute of Gerontology and Geriatrics, University of Perugia, Policlinico MontelucePadiglione E, Via Brunamonti 51, 06122 Perugia, Italy. E-mail:mecocci@unipg.it

*These two authors have contributed equally.

Accepted for publication February 6, 2008

\section{Significant outcomes}

- Neuropsychiatric symptoms were evaluated in a large sample of elderly subjects referred to a specialty clinical service. The most prevalent types of dementia (i.e. vascular dementia, dementia with Lewy bodies and Alzheimer's disease) were compared.

- Neuropsychiatric symptoms were evaluated with NPI, a validated, standardized and widely used instrument that was developed specifically for neuropsychiatric symptoms of dementia.

- Diagnosis of a degenerative type of dementia and its severity were independently associated with clinically relevant neuropsychiatric symptoms.

\section{Limitations}

- Neuropsychiatric symptoms were evaluated in a sample of elderly out-patients with low educational status who had mainly moderate stage dementia. Potential limitations are selection bias, reporter bias and whether results can be generalized.

- Residual confounders (e.g. use of psychotropic drugs, medical comorbidity and apolipoprotein E4 genotype) cannot be excluded, although most patients were not under treatment with antipsychotic drugs at the time of enrolment.

- Difference in prevalence of neuropsychiatric symptoms between data from this study and previous reports may depend upon sensitivity of instruments (NPI vs. other scales) and setting (clinical vs. population study). 


\section{Introduction}

Dementia is characterized by changes in cognitive, behavioural, psychological and functional capacities. Although cognitive deficits and decline in daily functioning are, according to the Diagnostic and Statistical Manual of Mental Disorders (DSM IVTR) (1), the main clinical features in the diagnosis of dementia, much evidence over the past decade has established neuropsychiatric symptoms (NPS) as fundamental clinical features (2). NPS, which include behavioural and psychological symptoms, are associated with poor prognosis (3), excess disability (4), impaired quality of life for subjects and carers (5), high levels of caregiver distress $(6,7)$, early institutionalization (3) and increased direct cost of care (8).

The frequency of NPS in dementia ranges from $25 \%$ to $80 \%(9,10)$, probably due to methodological differences and heterogeneous settings. However, previous studies have investigated mainly subjects with Alzheimer's disease (AD) (9, $11,12)$ and few have been conducted in subjects with vascular dementia (VaD) $(10,13-15)$ or dementia with Lewy bodies (DLB) $(16,17)$. According to previous findings, AD seems to be characterized by different behavioural syndromes $(12,18)$, which include prominent mood symptoms (i.e. depression, anxiety and apathy), psychotic disturbances (i.e. delusions and hallucinations), agitation-related symptoms (i.e. aggression and irritability) and/or symptoms of behavioural dyscontrol (i.e. euphoria, disinhibition, aberrant motor behaviour, sleep and appetite disturbances). Depression, anxiety and apathy, as well as irritability and psychotic disturbances, have been frequently described in subjects with $\mathrm{VaD}$ (19). Visual hallucinations, reported as diagnostic clinical features of DLB, are commonly observed during the course of the disease and may be the initial reason for referral; delusions, apathy, anxiety and depression are other NPS often associated with DLB $(20,21)$.

A previous report of NPS in dementias showed that $\mathrm{VaD}$ and $\mathrm{DLB}$ have a higher frequency of NPS than AD, although results are discordant and most refer to small case series $(13,16)$. Hence, the frequency of individual NPS in subjects with different types of dementia is still controversial.

Another important issue is the occurrence of NPS in the different stages of dementia $(9,11,22$, 23). Mood symptoms generally develop early in the course of the disease, while psychotic symptoms and agitation characterize the middle to late stages of dementia. Some NPS, such as depression or delusions and hallucinations, have been investigated in depth with specific instruments in subjects with dementia; others, like apathy or wandering, less so. In the last decade, numerous studies focused on developing standardized comprehensive scales for assessing NPS in dementia. Among them, the Neuropsychiatric Inventory (NPI) (24), appears to be a useful, reliable tool for assessing a wide spectrum of NPS and the Clinical Antipsychotic Trials of Intervention Effectiveness (CATIE) research program recently defined as clinically relevant a neuropsychiatric symptom that is severe enough to require treatment (NPI score 24) (25).

Aims of the study

We characterized the frequency and severity of neuropsychiatric symptoms using the NPI scale in a large sample of subjects with dementia referred to a clinical center and compared vascular dementia with the two most common forms of neurodegenerative dementias (i.e. dementia with Lewy bodies and Alzheimer's disease). Furthermore, we evaluated the impact of severity and type of dementia on expression of clinically relevant neuropsychiatric symptoms.

\section{Material and methods}

Subjects

From January 2001 to December 2005, 1235 subjects with dementia, all living at home, were consecutively seen at the Memory Clinic, Centre for the Study of Brain Aging and Senile Dementias, University of Perugia, Italy. The diagnostic work-up included a complete medical history, clinical and neurological examinations, laboratory testing, and neuroimaging with computed tomography or magnetic resonance imaging of the brain. All subjects or next-of-kin provided written informed consent as requested by the local ethical committee. All subjects underwent a complete neuropsychological evaluation. This included the Mini-Mental State Examination (MMSE) as a measure of global cognition followed by a specific battery of tests - the Babcock Story Recall, the Rey Auditory Verbal Learning test immediate and delayed recall, the Corsi Block Tapping test, the Token test, the Category Naming test, the Controlled Oral Word Association test, the Visual Search test and the Raven's Coloured Progressive Matrices. For each test, details on administration procedures and Italian normative data for score adjustment based on age, gender and education as 
well as normality cut-off scores $(\geq 95 \%$ of the lower tolerance limit of the normal population distribution) are available (26-28).

The severity of dementia was rated using the Clinical Dementia Rating scale (CDR) (29) whose score ranges from 0 (no cognitive decline) to 3 (severe dementia). Functional status was measured by Katz's index of activity of daily living (ADL) (30) whose score ranges from 0 (complete independence) to 6 (total dependence).

The diagnosis of dementia was made according to the DSM-IV-TR (1). AD was then diagnosed according to NINCDS-ADRDA criteria (31), VaD using the NINDS-AIREN criteria (32), DLB according to DLB consortium criteria (33), and frontal-temporal dementia (FTD) according to the Lund and Manchester criteria (34). Physicians who performed the diagnosis were blinded to the results of neuropsychological battery and the NPI. Dementia was first diagnosed after a discussion among geriatricians (M.C., E.M.) who examined the patients, after collecting the history, performing the clinical examination, evaluating the laboratory findings and the results of neuroimaging. Results of MMSE, CDR and ADL were also used. A further evaluation was then made by neurologists (R.M., R.C.) who were external to the recruitment process. If first and second diagnoses did not concur, data were re-examined independently by a senior geriatrician (P.M.) who made the final diagnosis. Kappa statistics evaluated the reliability of diagnosis between geriatricians and neurologists (35). As previously reported (36), combining NINCDS-ADRDA possible and probable AD categories $(n=68)$, there was a good interobserver agreement (kappa $=0.71)$.

One hundred and fifty-six subjects with dementia due to other general medical conditions (DSM-IVTR code 294.1), such as head trauma, severe metabolic or endocrine diseases, brain tumour, normal pressure hydrocephalus and other neurological disorders were excluded as well as subjects with a history of alcohol or substance abuse or dependence, with uncertain dementia, with delirium and with psychiatric disorders preceding the onset of dementia. In this latter group, we included subjects with a DSM-IV-TR diagnosis of mood disorders, psychotic disorders and personality disorders requiring chronic treatment with psychotropic drugs started before age 60 (1).

One hundred and thirty-two subjects, who were diagnosed as having possible $\mathrm{VaD}, \mathrm{DLB}, \mathrm{AD}$ and FTD were also excluded from the study. Of the remaining 947 subjects, 131 subjects fulfilled criteria for probable $\mathrm{VaD}, 100$ subjects for probable DLB, 690 for probable AD and 26 subjects for probable FTD. The latter group, being so small, was then excluded from data analyses, which refers to 921 subjects (Fig. 1).

Assessment of neuropsychiatric symptoms

NPS were evaluated by NPI (24), a fully structured caregiver interview with 12 sub-scales measuring the following symptoms: delusions, hallucinations, agitation, dysphoria, anxiety, apathy, irritability, euphoria, disinhibition, aberrant motor behaviour, sleeping and eating abnormalities. Each sub-scale has an entry question inquiring whether the disturbance had been present in the last month. If the answer is affirmative, the caregiver is asked to rate specific neurobehavioural symptoms within each sub-scale on a four-point frequency and on a three-point severity scale. Frequency and severity scores are multiplied for each sub-scale (composite score) and added together for the total NPI score. The composite score of each sub-scale ranges between 0 and 12, and the total composite score between 0 and 144 according to the severity and frequency of NPS. An NPI score of 4 or more for any symptom indicates clinical relevance and is considered entry criteria for treatment trials of dementia-associated NPS (25). The scale has a high level of internal consistency reliability, inter-rater reliability and the test-retest reliability, and has been validated in Italian subjects (37). As previously reported (37), inter-observer agreement on the presence/absence of clinically relevant NPS in subjects with probable $\mathrm{AD}(n=46)$ was almost perfect between the two raters who administered the NPI (percentage of agreement for each NPI sub-scale ranged from $87.6 \%$ to $95.5 \%$ ).

\section{Statistical analysis}

Demographic and clinical variables among groups were evaluated with one-way analysis of variance (ANOvA) with post-hoc Scheffe's test for multiple comparisons and chi-square analysis followed by Fisher's exact test, as needed. Frequencies of clinically relevant NPI domains (frequency $x$ severity score $\geq 4$ ) were also evaluated with chi-square analysis followed by Fisher's exact test. All tests were two tailed.

Logistic regression analysis assessed the association between each clinically relevant NPI symptom (dependent variable) and severity and type of dementia (independent variables).

Severity of dementia (CDR score) and type of dementia (VaD, DLB, AD) were analysed in two logistic regression models. The basic model was adjusted for age, gender, education and ADL score 


\section{Caputo et al.}

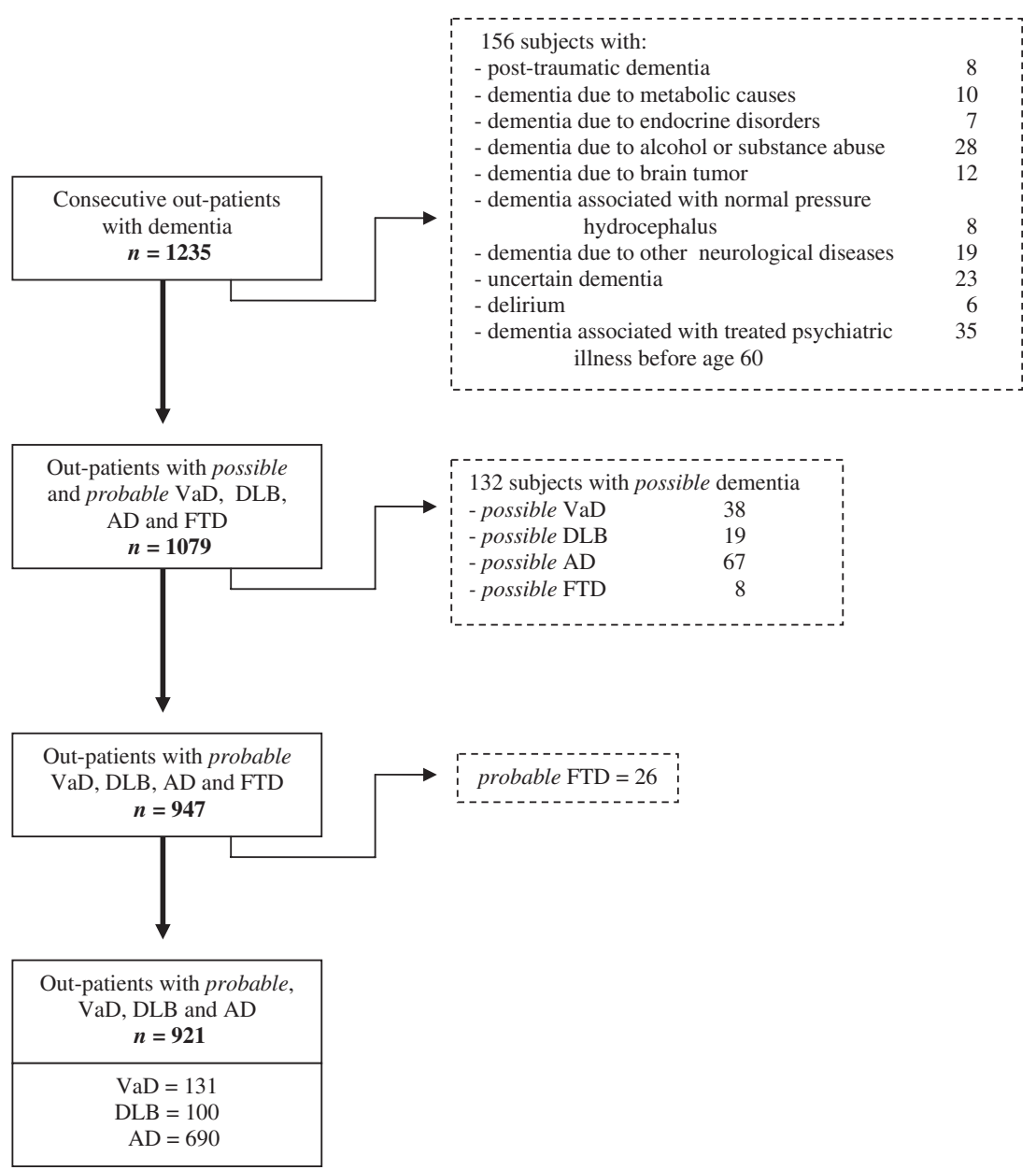

Fig. 1. Flow-chart diagram of formation of the study sample. (expressed as continuous variables); then it was adjusted for all these parameters plus either type of dementia (model 1) or CDR score (model 2). Results are presented as odds ratios (OR) with $95 \%$ confidence intervals $(95 \% \mathrm{CI})$. For all analyses, significance level was set at $P<0.05$. Statistical analysis was performed with SPss version 12.0 (SPSS, Inc., Chicago, IL, USA).

\section{Results}

ANOVA revealed a significant age-effect between groups. However, Scheffe's post-hoc analyses did not reveal statistical differences between groups at pair-comparison. Education, MMSE and CDR scores did not differ among groups. Subjects with $\mathrm{VaD}$ and DLB showed a significantly higher ADL score than those with AD. The percentage of women was significantly lower in $\mathrm{VaD}$ and DLB than in AD (Table 1).

Patients with $\mathrm{VaD}$ had a lower mean composite score in most NPI domains than DLB and AD, scoring significantly less in delusions, hallucinations, agitation/aggression, irritability and aberrant motor behaviour than patients with DLB and less in anxiety and aberrant motor behaviour than patients with AD. Subjects with DLB had significantly higher scores in agitation/aggression and disinhibition than those with $\mathrm{AD}$ and a higher total composite NPI score than subjects with $\mathrm{VaD}$ and AD (Table 2).

Clinically relevant NPS (NPI $\geq 4$ ) were common in all groups. One or more were present in $84.7 \%$ subjects with $\mathrm{VaD}, 96.0 \%$ with DLB and $90.6 \%$ with AD. Subjects with VaD showed a lower frequency of clinically relevant NPS, with significant differences for hallucinations (vs. DLB), agitation/aggression (vs. DLB and vs. AD), anxiety (vs. DLB and vs. AD), irritability (vs. DLB) and aberrant motor behaviour (vs. AD). Disinhibition was significantly more frequent in DLB than in AD (Table 3).

In $\mathrm{VaD}$, only frequency of clinically relevant apathy and aberrant motor behaviour increased significantly with disease progression. In DLB, the frequency of clinically relevant delusions, hallucinations, disinhibition and sleep disturbances was significantly higher in subjects with 
Neuropsychiatric symptoms in dementias

Table 1. Demographic and clinical data of subjects with $\mathrm{VaD}, \mathrm{DLB}$ and $\mathrm{AD}$

\begin{tabular}{lccccc}
\hline $\begin{array}{l}\text { Demographic and clinical } \\
\text { variables }\end{array}$ & $\begin{array}{c}\text { VaD } \\
(n=131)\end{array}$ & $\begin{array}{c}\text { DLB } \\
(n=100)\end{array}$ & $\begin{array}{c}\text { AD } \\
(n=690)\end{array}$ & P-Value & Post-hoc $^{*}$ \\
\hline Age (years) & $77.9(6.9)$ & $77.8(7.5)$ & $76.4(7.8)$ & 0.03 & \\
Education (years) & $5.6(4.2)$ & $5.1(3.4)$ & $5.1(3.7)$ & 0.31 & \\
ADL score & $2.9(1.9)$ & $2.9(1.9)$ & $2.1(1.9)$ & $<0.001$ & AD $<$ VaD, DLB \\
MMSE score & $15.0(6.2)$ & $14.0(7.4)$ & $14.7(6.5)$ & 0.51 & \\
CDR score & $2.0(0.6)$ & $2.1(0.7)$ & $2.1(0.7)$ & 0.36 & \\
Women, $n(\%)$ & $72(55.0)$ & $51(51.0)$ & $487(70.6)$ & $<0.001$ & VaD, DLB < AD \\
Dementia severity (CDR score), $n(\%):$ & & & & 0.22 & \\
$\quad$ CDR 1 (mild) & $27(20.6)$ & $21(21.0)$ & $138(20)$ & & \\
CDR 2 (moderate) & $79(60.3)$ & $47(47.0)$ & $376(54.5)$ & & \\
CDR 3 (severe) & $25(19.1)$ & $32(32.0)$ & $176(25.5)$ & & \\
\hline
\end{tabular}

VaD, vascular dementia; DLB, dementia with Lewy bodies; AD, Alzheimer disease; ADL, activity of daily living; MMSE, mini-mental state examination; CDR, clinical dementia rating.

Data are expressed as mean (SD) unless otherwise specified.

${ }^{*}$ ANova with post-hoc Scheffe's test (ADL) or chi-square with Fisher's exact test (gender).

\begin{tabular}{lccccc}
\hline NPI item & $\operatorname{VaD}(n=131)$ & $\mathrm{DLB}(n=100)$ & $\mathrm{AD}(n=690)$ & $P$-value & Post-hoc $^{*}$ \\
\hline Delusions & $1.2(2.5)$ & $2.2(3.3)$ & $1.7(2.9)$ & 0.04 & $\mathrm{VaD}<\mathrm{DLB}$ \\
Hallucinations & $0.6(1.4)$ & $1.4(2.7)$ & $1.0(2.3)$ & 0.02 & $\mathrm{VaD}<\mathrm{DLB}$ \\
Agitation /aggression & $2.3(2.8)$ & $3.8(4.0)$ & $2.9(3.3)$ & 0.002 & $\mathrm{VaD}, \mathrm{AD}<\mathrm{DLB}$ \\
Depression & $3.1(3.4)$ & $3.0(3.2)$ & $3.2(3.5)$ & 0.89 & \\
Anxiety & $2.1(2.8)$ & $3.1(3.3)$ & $3.0(3.2)$ & 0.01 & $\mathrm{VaD}<\mathrm{AD}$ \\
Euphoria & $0.4(1.6)$ & $0.4(1.2)$ & $0.3(1.2)$ & 0.80 & \\
Apathy & $3.8(3.4)$ & $4.8(4.0)$ & $4.1(3.9)$ & 0.13 & \\
Disinhibition & $0.9(2.2)$ & $1.4(2.6)$ & $0.8(2.0)$ & 0.02 & $\mathrm{AD}<\mathrm{DLB}$ \\
Irritability & $2.2(2.9)$ & $3.4(3.4)$ & $2.8(3.1)$ & 0.005 & $\mathrm{VaD}<\mathrm{DLB}$ \\
Aberrant motor behaviour & $1.3(3.0)$ & $2.4(3.6)$ & $2.2(3.4)$ & 0.01 & $\mathrm{VaD}<\mathrm{DLB}, \mathrm{AD}$ \\
Sleep & $2.0(3.0)$ & $2.6(3.6)$ & $2.0(3.2)$ & 0.22 & \\
Eating & $1.2(2.6)$ & $1.8(3.3)$ & $1.5(3.0)$ & 0.28 & \\
Total NPI & $20.0(16.0)$ & $30.4(21.1)$ & $24.3(18.6)$ & $<0.001$ & $\mathrm{VaD}, \mathrm{AD}<\mathrm{DLB}$ \\
\hline
\end{tabular}

VaD, vascular dementia; DLB, dementia with Lewy bodies; AD, Alzheimer disease; NPI, neuropsychiatric inventory. Data are expressed as mean (SD) unless otherwise specified.

${ }^{*}$ Anova with post-hoc Scheffe's test.

\begin{tabular}{lccccc}
\hline NPI item & $\operatorname{VaD}(n=131)$ & $\mathrm{DLB}(n=100)$ & $\mathrm{AD}(n=690)$ & $P$-value & $\begin{array}{c}\text { Two-by-two } \\
\text { comparison* }\end{array}$ \\
\hline Delusions & $22(16.8)$ & $27(27.0)$ & $156(22.6)$ & 0.17 & \\
Hallucinations & $10(7.6)$ & $18(18.0)$ & $87(12.6)$ & 0.06 & $\mathrm{VaD}<\mathrm{DLB}$ \\
Agitation/aggression & $38(29.0)$ & $48(48.0)$ & $286(41.4)$ & 0.008 & $\mathrm{VaD}<\mathrm{DLB}, \mathrm{AD}$ \\
Depression & $57(43.5)$ & $38(38.0)$ & $278(40.3)$ & 0.68 & \\
Anxiety & $40(30.5)$ & $46(46.0)$ & $289(41.9)$ & 0.03 & $\mathrm{VaD}<\mathrm{DLB}, \mathrm{AD}$ \\
Euphoria & $5(3.8)$ & $5(5.0)$ & $29(4.2)$ & 0.90 & \\
Apathy & $72(55.0)$ & $60(60.0)$ & $354(51.3)$ & 0.23 & \\
Disinhibition & $16(12.2)$ & $20(20.0)$ & $66(9.6)$ & 0.007 & $\mathrm{AD}<\mathrm{DLB}$ \\
Irritability & $44(33.6)$ & $51(51.0)$ & $273(39.6)$ & 0.02 & $\mathrm{VaD}<\mathrm{DLB}$ \\
Aberrant motor behaviour & $22(16.8)$ & $30(30.0)$ & $218(31.6)$ & 0.003 & $\mathrm{VaD}<\mathrm{AD}$ \\
Sleep & $35(26.7)$ & $32(32.0)$ & $174(25.2)$ & 0.35 & \\
Eating & $20(15.3)$ & $24(24.0)$ & $132(19.1)$ & 0.25 & \\
\hline
\end{tabular}

VaD, vascular dementia; DLB, dementia with Lewy bodies; AD, Alzheimer disease; NPI, neuropsychiatric inventory. Data are expressed as $n(\%)$ unless otherwise specified.

${ }^{*}$ Chi-square with Fisher's exact test. moderate-severe dementia than in those with mild dementia. In AD, except for anxiety and depression, the frequency of clinically relevant NPS, increased with severity of dementia. Anxiety tended to decrease as dementia became more severe; depression was significantly more frequent in moderate than in mild or severe AD (Table 4).

Logistic regression analysis with mild dementia (CDR 1) as reference group showed that moderate dementia (CDR 2) was significantly 


\section{Caputo et al.}

Table 4. Frequency of clinically relevant NPI symptoms (frequency $\times$ severity $\geq 4$ ) according to dementia severity (CDR score) in subjects with VaD, DLB and AD

\begin{tabular}{|c|c|c|c|c|c|c|c|c|c|c|c|c|c|c|c|}
\hline \multirow[b]{2}{*}{ NPI item } & \multicolumn{5}{|c|}{ CDR score in $\mathrm{VaD}, \%$} & \multicolumn{5}{|c|}{ CDR score in DLB, \% } & \multicolumn{5}{|c|}{ CDR score in $A D, \%$} \\
\hline & $\begin{array}{c}1 \\
(n=27)\end{array}$ & $\begin{array}{c}2 \\
(n=79)\end{array}$ & $\begin{array}{c}3 \\
(n=25)\end{array}$ & $P$-value & $\begin{array}{c}\text { Two- } \\
\text { by-two } \\
\text { comparison* }\end{array}$ & $\begin{array}{c}1 \\
(n=21)\end{array}$ & $\begin{array}{c}2 \\
(n=47)\end{array}$ & $\begin{array}{c}3 \\
(n=32)\end{array}$ & $P$-value & $\begin{array}{c}\text { Two- } \\
\text { by-two } \\
\text { comparison* }\end{array}$ & $\begin{array}{c}1 \\
(n=138)\end{array}$ & $\begin{array}{c}2 \\
(n=376)\end{array}$ & $\begin{array}{c}3 \\
(n=176)\end{array}$ & $P$-value & $\begin{array}{c}\text { Two- } \\
\text { by-two } \\
\text { comparison* }\end{array}$ \\
\hline Delusions & 14.8 & 16.5 & 20.0 & 0.88 & & 14.3 & 21.3 & 43.8 & 0.03 & $1,2<3$ & 14.5 & 19.9 & 34.7 & $<0.001$ & $1,2<3$ \\
\hline Hallucinations & 0 & 8.9 & 12.0 & 0.22 & & 0 & 19.1 & 28.1 & 0.03 & $1<2,3$ & 4.3 & 11.2 & 22.2 & $<0.001$ & $1<2<3$ \\
\hline $\begin{array}{l}\text { Agitation/ } \\
\text { aggression }\end{array}$ & 25.9 & 27.8 & 36.0 & 0.68 & & 42.9 & 44.7 & 56.3 & 0.52 & & 33.3 & 39.6 & 51.7 & 0.003 & $1,2<3$ \\
\hline Depression & 33.3 & 49.4 & 36.0 & 0.25 & & 38.1 & 44.7 & 2.1 & 0.33 & & 31.2 & 45.7 & 35.8 & 0.004 & $1,3<2$ \\
\hline Anxiety & 25.9 & 31.6 & 32.0 & 0.84 & & 47.6 & 48.9 & 40.6 & 0.76 & & 44.2 & 43.9 & 35.8 & 0.17 & \\
\hline Euphoria & 3.7 & 2.5 & 8.0 & 0.46 & & 0 & 6.4 & 6.3 & 0.50 & & 2.9 & 2.4 & 9.1 & 0.001 & $1,2<3$ \\
\hline Apathy & 25.9 & 60.8 & 68.0 & 0.002 & $1<2,3$ & 61.9 & 63.8 & 53.1 & 0.62 & & 38.4 & 52.4 & 59.1 & 0.001 & $1<3$ \\
\hline Disinhibition & 3.7 & 11.4 & 24.0 & 0.08 & & 0 & 14.9 & 40.6 & 0.001 & $1,2<3$ & 5.8 & 8.2 & 15.3 & 0.007 & $1,2<3$ \\
\hline Irritability & 37.0 & 31.6 & 36.0 & 0.84 & & 47.6 & 53.2 & 50 & 0.91 & & 38.4 & 35.6 & 48.9 & 0.01 & $2<3$ \\
\hline $\begin{array}{l}\text { Aberrant motor } \\
\text { behaviour }\end{array}$ & 7.4 & 12.7 & 40.0 & 0.002 & $1,2<3$ & 19.0 & 31.9 & 34.4 & 0.46 & & 17.4 & 26.9 & 52.8 & $<0.001$ & $1<2<3$ \\
\hline Sleep & 14.8 & 26.6 & 40.0 & 0.12 & & 4.8 & 34.0 & 49.9 & 0.005 & $1<2,3$ & 17.4 & 23.7 & 34.7 & 0.001 & $1,2<3$ \\
\hline Eating & 11.1 & 13.9 & 24.0 & 0.38 & & 23.8 & 14.9 & 37.5 & 0.07 & & 10.1 & 19.4 & 25.6 & 0.003 & $1<2,3$ \\
\hline
\end{tabular}

VaD, vascular dementia; DLB, dementia with Lewy bodies; AD, Alzheimer disease; NPI, neuropsychiatric inventory; CDR, clinical dementia rating.

${ }^{*}$ Chi-square with Fisher's exact test.

\begin{tabular}{|c|c|c|c|c|}
\hline \multirow[b]{2}{*}{ NPI item } & \multicolumn{2}{|c|}{ CDR score (model 1) } & \multicolumn{2}{|c|}{ Diagnosis (model 2) } \\
\hline & CDR 2 vs. CDR 1 & CDR 3 vs. CDR 1 & DLB vs. VaD & $A D$ vs. VaD \\
\hline Delusions & $1.2(0.7-1.9)$ & $1.9(1.1-3.3)$ & $1.7(0.9-3.3)$ & $1.7(1.0-2.9)$ \\
\hline Hallucinations & $3.0(1.3-7.3)$ & $4.8(1.9-12.1)$ & $2.5(1.1-5.9)$ & $2.2(1.1-4.5)$ \\
\hline Agitation/aggression & $1.0(0.7-1.5)$ & $1.4(0.9-2.2)$ & $2.2(1.2-3.8)$ & $2.1(1.3-3.1)$ \\
\hline Depression & $1.5(1.1-2.2)$ & $0.9(0.6-1.4)$ & $0.8(0.5-1.5)$ & $0.9(0.6-1.3)$ \\
\hline Anxiety & $0.9(0.6-1.2)$ & $0.6(0.4-0.97)$ & $2.0(1.2-3.5)$ & $1.6(1.1-2.5)$ \\
\hline Euphoria & $0.8(0.3-2.4)$ & $1.9(0.6-5.7)$ & $1.1(0.3-3.9)$ & $1.1(0.4-3.2)$ \\
\hline Apathy & $1.8(1.2-2.6)$ & $1.7(1.1-2.7)$ & $1.2(0.7-2.1)$ & $1.0(0.7-1.5)$ \\
\hline Disinhibition & $1.8(0.8-3.8)$ & $3.4(1.5-7.8)$ & $1.6(0.8-3.4)$ & $0.9(0.5-1.7)$ \\
\hline Irritability & $0.8(0.5-1.1)$ & $1.0(0.7-1.6)$ & $1.9(1.1-3.3)$ & $1.5(1.0-2.2)$ \\
\hline Aberrant motor behaviour & $1.5(0.95-2.4)$ & $3.5(2.1-5.9)$ & $2.0(1.0-3.8)$ & $2.7(1.6-4.5)$ \\
\hline Sleep & $1.2(0.7-2.0)$ & $1.4(0.8-2.4)$ & $1.2(0.7-2.3)$ & $1.4(0.8-2.2)$ \\
\hline Eating & $1.3(0.8-2.2)$ & $1.5(0.8-2.6)$ & $1.8(0.9-3.5)$ & $1.7(1.0-2.9)$ \\
\hline
\end{tabular}

Table 5. Association between clinically relevant NPI symptoms (frequency $\times$ severity $\geq 4$ ) and, respectively, dementia severity (CDR score) or dementia type

Values are given as odds ratio $(95 \% \mathrm{Cl})$.

$\mathrm{VaD}$, vascular dementia; DLB, dementia with Lewy bodies; AD, Alzheimer disease; NPI, neuropsychiatric inventory: CDR, clinical dementia rating. Logistic regression analyses were adjusted for: age, gender, education, ADL score and diagnosis (model 1); age, gender, education, ADL score and CDR score (model 2). Statistically significant results are reported in bold type.

associated with clinically relevant hallucinations, depression and apathy. Severe dementia (CDR 3) was significantly associated with clinically relevant delusions, hallucinations, apathy, disinhibition and aberrant motor behaviour (Table 5).

Logistic regression analysis with $\mathrm{VaD}$ as reference group showed DLB was significantly associated with clinically relevant hallucinations, agitation/aggression, anxiety, irritability, and aberrant motor behaviour and AD was significantly associated with clinically relevant delusions, hallucinations, agitation/aggression, anxiety, irritability, aberrant motor behaviour and eating disturbances (Table 5).

\section{Discussion}

The present study provides evidence that frequency of clinically relevant NPS varies with the type and severity of dementia, which were independently associated with NPS expression. In this study, subjects with DLB showed the highest severity and frequency of clinically relevant NPS. Unlike VaD, neurodegenerative dementias, particularly $\mathrm{AD}$, were associated with more frequent NPS as cognitive impairment worsened, thus confirming previous results $(38,39)$.

To our knowledge, this is the first study to evaluate NPS in a large sample of subjects with the 
three most common types of dementia using the NPI, a comprehensive, standardized widely used instrument (24).

Regardless of the type of dementia, these data confirm that NPS are very common in subjects with dementia who have been referred to a memory clinic. Concurring with previous reports on outpatients $(11,38,40)$, at least one NPS was found in about $90 \%$ of cases. Apathy was extremely frequent, being present in over $50 \%$ of subjects with any type of dementia, thereby supporting previous observations in VaD (19), DLB (21) and AD (9-11).

In this study, $\mathrm{VaD}$ was associated with a lower mean NPI score and fewer clinically relevant NPI symptoms than DLB and AD. Unlike these results, Lyketsos et al. (10) and Fuh et al. (41) found few differences in the frequency and severity of NPS (except for delusions and depression) in large samples of subjects with $\mathrm{VaD}$ and AD. Even though other studies $(13,14,39-41)$ have reported depression, anxiety and/or apathy are more frequent in subjects with $\mathrm{VaD}$, this study found a significantly lower frequency of anxiety in $\mathrm{VaD}$ than in DLB and AD but no differences in the frequency of depression and apathy. Concerning degenerative dementias, the severity and frequency of most disturbances were particularly evident in subjects with DLB, as previously reported $(20,21)$. They were more evident than in patients with $\mathrm{VaD}$ and, except for disinhibition and agitation, tended to overlap with the pattern observed in AD. In $\mathrm{AD}$, the spectrum of clinically relevant NPS was similar to previous reports (9-11): apathy was the most frequent behavioural disturbance, with a high prevalence of anxiety, agitation, depression; the least common NPS was euphoria.

Severity of dementia was not always associated with worsening NPS. In VaD, severity of disease did not impact upon frequency of NPS, except for apathy and aberrant motor behaviour, thereby confirming other reports $(38,39)$. However, discrepancies in this study that reports that psychosis, depression and anxiety were more common in moderate and severe $\operatorname{VaD}(13,14)$, may in part be related to the relatively small sample size of the $\mathrm{VaD}$ group and to the focus of this study on clinically relevant NPS. On the contrary, in DLB frequency of delusions, hallucinations, disinhibition and sleep problems increased with severity of dementia. These data support a previous study reporting that in DLB NPS are related, although weakly, to severity of dementia (21). Interestingly, in the mild stage of DLB visual hallucinations, which are one of its core features (33), did not reach the clinically relevant threshold. Unlike these findings, Ballard et al. (17), reported depression, delusion, hallucination and delusional misidentification were equally common in different stages of DLB. Again, discrepancies are probably due to our decision to focus on clinically relevant disturbances. Indeed, when hallucinations - one of the core symptoms of DLB - were considered only as 'present' (NPI score $\geq 1$ ), they were found in $19 \%$ of patients with DLB at mild, in $34 \%$ at moderate and $41 \%$ at severe stage of dementia. In patients with $\mathrm{AD}$, severity of dementia was most often associated with increased frequency of clinically relevant NPS, as reported elsewhere $(11,22,23$, 42). Independently of disease severity and other confounders (such as age, gender, disability, that influence NPS) $(22,41,43)$, suffering from DLB or AD roughly doubles the risk of presenting clinically relevant NPS compared with VaD.

Discrepancies in frequency rates and severity of NPS across different types and stages of dementia may reflect differences in methodological and statistical approaches. First, they may be due to the differential sensitivity of instruments since other studies used scales other than NPI or CDR to evaluate NPS and dementia severity $(14,17,39)$. Second, this research focused on clinically relevant symptoms, excluding subjects with very mild NPS, so as to obtain robust observations on subjects who often need not only pharmacological but also non-pharmacological therapies. Third, differences in demographics could account for discrepancies since the study sample included elderly subjects, with a low-educational status and who were mainly at moderate stage of dementia. Lastly, the different study-setting (e.g. hospital based vs. population based) may explain the differences observed in the present study from those obtained at population level (10).

Although psychological and social factors contribute to the onset and persistence of NPS in dementia (44), the different profiles of clinically relevant NPS which emerged in vascular and neurodegenerative dementias may reflect the involvement of specific brain regions. For example, data collected using voxel-based morphometry showed that in neurodegenerative dementias, the presence of apathy was independently associated with tissue loss in the right ventromedial superior frontal gyrus, disinhibition with tissue loss in the right ventromedial prefrontal cortex, while aberrant motor behaviour with tissue loss in the right anterior cingulate cortex and left premotor cortex (45). Furthermore, well-formed visual hallucinations in subjects with DLB have been related to density of Lewy bodies in the amygdala and parahippocampus (46). In patients with AD 
agitation and aberrant motor behaviour were associated with a greater neurofibrillary tangle burden in the orbital-frontal cortex and apathy in the anterior cingulate area (47).

On the basis of previous (41) and our results, it could be suggested that NPS in VaD probably reflect the variability of the location and the extension of ischaemic damage (cortical vs. subcortical $\mathrm{VaD}$ ). On the contrary, these and previous data (41) propose that NPS severity in neurodegenerative dementias, particularly $\mathrm{AD}$, parallel the progressive atrophy of fronto-temporo-parietal association cortices (48).

Strengths of this study include its large sample size, the use of a standardized diagnostic procedure and adjustment for multiple potential confounders.

However, some methodological issues deserve mentioning. Caution is needed in extending these findings to the general population because the study sample was recruited in a specialized clinical setting, which might have introduced a selection bias. For example, we can hypothesize that subjects with $\mathrm{VaD}$ are less represented in our sample compared with some epidemiological studies because these patients have often a severe motor impairment and, consequently, major difficulties of being observed in an outpatient setting. Furthermore, due to the fact that NPI is a caregiver-based interview, reporter bias cannot be excluded. Dementia was diagnosed according to clinical criteria, with inevitably some uncertainty about the rate of misclassification. Without post-mortem confirmation, a standardized diagnostic procedure was used with a good between observer agreement, and clinical diagnoses were supported by brain imaging assessments. However, it must be acknowledged that current criteria for DLB have low sensitivity when Lewy bodies coexist with the pathological abnormalities of Alzheimer disease (49). Although analyses were adjusted for major potential confounders, residual confounding (medical comorbidity, use of psychotropic drugs and apolipoprotein E4 genotype) (50-53) cannot be excluded. Concerning the use of psychotropic drug, however, all patients included in the study were at their first assessment and the majority of cases were untreated. Finally, the cross-sectional design of the study may not be optimal since behavioural disturbances can fluctuate and may not be present at every examination (54). Moreover, due to the cross-sectional study design, a relationship between the type and severity of dementia and NPS expression can only be hypothesized.

In conclusion, the results of this study suggest that different types of dementias show characteristic behavioural profiles. In particular, NPS appear to be more frequent and severe in degenerative than in vascular dementia. The frequency of potentially treatable NPS increases with disease severity in degenerative dementias, particularly in AD. Longitudinal data on large samples of subjects are needed to evaluate the prognostic role of NPS in different types of dementia.

\section{Acknowledgement}

The authors thank Geraldine A Boyd BA (hons), D. Lit for English revision.

\section{References}

1. American Psychiatric Association. Diagnostic and Statistical Manual of Mental Disorders. IV-TR ed. Washington, DC: American Psychological Association, 2000.

2. Mc Keith I, Cummings J. Behavioral changes and psychological symptoms in dementia disorders. Lancet Neurol 2005;4:735-742.

3. Stern Y, Tang MX, Albert MS et al. Predicting time to nursing home care and death in individuals with Alzheimer disease. JAMA 1997;277:806-812.

4. Norton LE, Malloy PF, Salloway S. The impact of behavioral symptoms on activities of daily living in subjects with dementia. Am J Geriatr Psychiatry 2001;9:41-48.

5. Gonzalez-Salvador T, Lyketsos CG, Baker A et al. Quality of life of subjects with dementia in long-term care. Int $\mathbf{J}$ Geriatr Psychiatry 2000;15:181-189.

6. Rinaldi P, Spazzafumo L, Mastriforti R et al. Predictors of high level of burden and distress in caregivers of demented subjects: results of an Italian multicenter study. Int $\mathbf{J}$ Geriatr Psychiatry 2005;20:168-174.

7. Sink KM, Covinsky KE, Barnes DE, Newcomer RJ, Yaffe K. Caregiver characteristics are associated with neuropsychiatric symptoms of dementia. J Am Geriatr Soc 2006; 54:796-803.

8. Murman Dl, Chen Q, Powell MC, Kuo SB, Bradley CJ, Colenda CC. The incremental direct costs associated with behavioral symptoms in AD. Neurology 2002;59:17211729.

9. Mega MS, Cummings JL, Fiorello T, Gornbein J. The spectrum of behavioral changes in Alzheimer's disease. Neurology 1996;46:130-135.

10. Lyketsos CG, Steinberg M, Tschanz JT, Norton MC, StefFENS DC, BReitner JC. Mental and behavioral disturbances in dementia: findings from the Cache County Study on Memory in Aging. Am J Psychiatry 2000;157:708-714.

11. Craig D, Mirakhur A, Hart DJ, Mcilroy SP, Passmore AP. A cross-sectional study of neuropsychiatric symptoms in 435 patients with Alzheimer's disease. Am J Geriatr Psychiatry 2005;13:460-468.

12. Hollingworth P, Hamshere ML, Moskvina V et al. Four components describe behavioral symptoms in 1,120 individuals with late-onset Alzheimer's disease. J Am Geriatr Soc 2006;54:1348-1354.

13. Aharon-Peretz J, Kliot D, Tomer R. Behavioral differences between white matter lacunar dementia and Alzheimer's disease: a comparison on the neuropsychiatric inventory. Dement Geriatr Cogn Disord 2000;11:294-298.

14. Ballard C, Neill D, O'brien J et al. Anxiety, depression and psychosis in vascular dementia: prevalence and associations. J Affect Disord 2000;59:97-106. 
15. Leroi I, Voulgari A, Breitner JC, Lyketsos CG. The epidemiology of psychosis in dementia. Am J Geriatr Psychiatry 2003;11:83-91.

16. Rockwell E, Choure J, Galasko D, Olichney J, Jeste D. Psychopathology at initial diagnosis in dementia with Lewy bodies versus Alzheimer disease: comparison of matched groups with autopsy-confirmed diagnoses. Int $\mathbf{J}$ Geriatr Psychiatry 2000;15:819-823.

17. Ballard C, Holmes C, Mckeith I et al. Psychiatric morbidity in dementia with Lewy bodies: a prospective clinical and neuropathological comparative study with Alzheimer's disease. Am J Psychiatry 1999;156:1039-1045.

18. Aalten P, De Vugt ME, Jaspers $\mathrm{N}$ et al. The course of neuropsychiatric symptoms in dementia. Part II: relationships among behavioral sub-syndromes and the influence of clinical variables. Int J Geriatr Psychiatry 2005;20:531536.

19. O'brien JT, Erkinjuntti T, Reisberg B et al. Vascular cognitive impairment. Lancet Neurol 2003;2:89-98.

20. Mckeith I, Mintzer J, Aarsland D et al. Dementia with Lewy bodies. Lancet Neurol 2004;3:19-28.

21. Del Ser T, Mckeith I, Anand R, Cicin-Sain A, Ferrara R, SPIEgEL R. Dementia with Lewy bodies: findings from an international multicentre study. Int $\mathbf{J}$ Geriatr Psychiatry 2000;15:1034-1045.

22. Harwood DG, Barker WW, Ownby RL, Duara R. Relationship of behavioral and psychological symptoms to cognitive impairment and functional status in Alzheimer's disease. Int J Geriatr Psychiatry 2000;15:393-400.

23. Lopez OL, Becker JT, Sweet RA et al. Psychiatric symptoms vary with the severity of dementia in probable Alzheimer's disease. J Neuropsychiatry Clin Neurosci 2003;15:346-353.

24. Cummings JL, Mega M, Gray K, Rosenberg-Thompson S, CARusi DA, Gornbein J. The neuropsychiatric inventory: comprehensive assessment of psychopathology in dementia. Neurology 1994;44:2308-2314.

25. Schneider LS, TARIOT PN, LyKetsos CG et al. National Institute of Mental Health Clinical Antipsychotic Trials of Intervention Effectiveness (CATIE): Alzheimer disease trial methodology. Am J Geriatr Psychiatry 2001;9:346360.

26. Magni E, Binetti G, Bianchetti A, Rozzini R, Trabucchi M. Mini-mental state examination: a normative study in italian elderly population. Eur J Neurol 1996;3:1-5.

27. Spinnler H, Tognoni G, Italian Group on the NeuropsychoLogical Study of Ageing. Italian standardization and classification of neuropsychological tests. Ital J Neurol Sci 1987;6(suppl 8):1-120.

28. Carlesimo GA, Caltagirone C, Gainotti G. The Mental Deterioration Battery: normative data, diagnostic reliability and qualitative analyses of cognitive impairment. The Group for the Standardization of the Mental Deterioration Battery. Eur Neurol 1996;36:378-384.

29. Morris JC. Clinical dementia rating: a reliable and valid diagnostic and staging measure for dementia of the Alzheimer type. Int Psychogeriatr 1997;9:173-176.

30. Katz S, Downs TD, Cash HR, Grotz RC. Index of activities of daily living. Gerontologist 1970;10:20-30.

31. Mckhann G, Drachman D, Folstein M et al. Clinical diagnosis of Alzheimer's disease: report of the NINCDS-ADRDA Work Group under the auspices of Department of Health and Human Services Task Force on Alzheimer's Disease. Neurology 1984;34:939-944.

32. Román GC, Tatemichi TK, ERkinjuntti T et al. Vascular dementia: diagnostic criteria for research studies. Report of the NINDS-AIREN International Workshop. Neurology 1993;43:250-260.
33. Mскеith IG, Galasko D. Consensus guidelines for the clinical and pathologic diagnosis of dementia with Lewy bodies (DLB): report of the consortium on DLB international workshop. Neurology 1996;47:1113-1124.

34. The Lund and Manchester Groups. Clinical and neuropathological criteria for frontotemporal dementia. J Neurol Neurosurg Psychiatry 1994;57:416-418.

35. Landis JR, Косн GG. The measurement of observer agreement for categorical data. Biometrics 1977;33:159-174.

36. Lopez OL, Litvan I, Catt KE et al. Accuracy of four clinical diagnostic criteria for the diagnosis of neurodegenerative dementias. Neurology 1999;53:1292-1299.

37. Binetti G, Mega MS, Magni E E et.al. Behavioral disorders in Alzheimer disease: a transcultural perspective. Arch Neurol 1998;55:539-544.

38. Kurita A, Blass JP, Nolan Ka, Black RS, Thaler HT. Relationship between cognitive status and behavioral symptoms in Alzheimer's disease and mixed dementia. J Am Geriatr Soc 1993;41:732-736.

39. Kim JM, Lyons D, Shin IS, Yoon JS. Differences in the behavioral and psychological symptoms between Alzheimer's disease and vascular dementia: are the different pharmacologic treatment strategies justifiable? Hum Psychopharmacol 2003;18:215-220.

40. Peters KR, Rockwood K, Black SE et al. Characterizing neuropsychiatric symptoms in subjects referred to dementia clinics. Neurology 2006;66:523-528.

41. Fun JL, Wang SJ, Cummings JL. Neuropsychiatric profiles in subjects with Alzheimer's disease and vascular dementia. J Neurol Neurosurg Psychiatry 2005;76:1337-1341.

42. Weiner MF, Hynan LS, Bret ME, White C 3Rd. Early behavioral symptoms and course of Alzheimer's disease. Acta Psychiatr Scand 2005;111:367-371.

43. Отt BR, Lapane KL, Gambassi G. Gender differences in the treatment of behavior problems in Alzheimer's disease. SAGE Study Group. Systemic Assessment of Geriatric drug use via Epidemiology. Neurology 2000;54:427-432.

44. Aarsland D, Sharp S, Ballard C. Psychiatric and behavioral symptoms in Alzheimer's disease and other dementias: etiology and management. Curr Neurol Neurosci Rep 2005;5:345-354.

45. Rosen HJ, Allison SC, Schauer GF, Gorno-Tempini ML, Weiner MW, Miller BL. Neuroanatomical correlates of behavioral disorders in dementia. Brain 2005;128: 2612-2625.

46. Harding AJ, Broe GA, Halliday GM. Visual hallucinations in Lewy body disease relate to Lewy bodies in the temporal lobe. Brain 2002;125:391-403.

47. Tekin S, Mega MS, Masterman DM et al. Orbitofrontal and anterior cingulate cortex neurofibrillary tangle burden is associated with agitation in Alzheimer disease. Ann Neurol 2001;49:355-361.

48. Masdeu JC, Zubieta JL, Arbizu J. Neuroimaging as a marker of the onset and progression of Alzheimer's disease. J Neurol Sci 2005;236:55-64.

49. Lopez OL, Becker JT, Kaufer DI et al. Research evaluation and prospective diagnosis of dementia with Lewy bodies. Arch Neurol 2002;59:43-46.

50. Tekin S, Fairbanks LA, O'connor S, Rosenberg S, Cummings JL. Activities of daily living in Alzheimer's disease: neuropsychiatric, cognitive, and medical illness influences. Am J Geriatr Psychiatry 2001;9:81-86.

51. Selbaek G, Kirkevold O, Engedal K. The prevalence of psychiatric symptoms and behavioral disturbances and the use of psychotropic drugs in Norwegian nursing homes. Int J Geriatr Psychiatry 2007;22:843-849. 


\section{Caputo et al.}

52. Monastero R, Mariani E, Camarda C et al. Association between apolipoprotein $\mathrm{E}$ epsilon4 allele and apathy in probable Alzheimer's disease. Acta Psychiatr Scand 2006;113:59-63.

53. Scarmeas N, Brandt J, Albert M et al. Association between the APOE genotype and psychopathologic symptoms in Alzheimer's disease. Neurology 2002;58:1182-1188.
54. Aalten P, De Vugt Me, Jaspers N, Jolles J, Verhey FR. The course of neuropsychiatric symptoms in dementia. Part I: findings from the two-year longitudinal Maasbed study. Int J Geriatr Psychiatry 2005;20:523-530. 